\title{
The Principles and Influence of Negative Stereotypes
} -Analysis with Examples

\author{
Peige Wang ${ }^{1, *}$ \\ ${ }^{1}$ Business School, Northeast Normal University, Changchun, Jilin Province, China, 130000 \\ *Corresponding author. Email: wangpg690@nenu.edu.cn
}

\begin{abstract}
For a long time, stereotypes have been the focus of attention of the public and psychologists, and different scientists have put forward different theories about this phenomenon. The purpose of this article is to sort out the research objects that have always been focused on, from one-dimensional negative stereotypes to multi-dimensional stereotype content models (SCM), from female groups and minority ethnic groups to a wide range of social groups. At the same time, this article explains the harm of stereotypes to society, such as the disadvantaged position of women in school and work, and gives some suggestions related to education and fair promotion system to reduce the impact of stereotypes on society.
\end{abstract}

Keywords: stereotype, gender stereotype, stereotype threat, stereotype lift, stereotype content model (SCM)

\section{INTRODUCTION}

Stereotypes are common across cultures, and we have many pieces of evidence to prove the existence of this phenomenon: Bolukbasiand his colleagues found that although the definition of programmer itself has nothing to do with gender, in the news corpus, programmers are more connected with men0; Carr found in the long-term follow-up research that men $(21 \%)$ are more likely than women $(10 \%)$ to become leaders and stay in their positions for longer periods in the medical field0. Although stereotypes have been widely discussed and used in psychology community and news reports, the public's basic understanding of stereotypes still needs to be strengthened. In fact, these deep-rooted and subconscious stereotypes lead to behaviors that require public social discussions and repeated reminders from others to make the parties aware of the existence of these behaviors. Through reading a variety of papers, the author summarized the objective observations and theoretical analysis of scientists on stereotypes, sorted out the classic phenomena and more mature theories in this field, and explained the widespread but negative impact of this phenomenon on society. The author tried to use this method to increase the public's awareness of stereotypes, and proposed some measures to eliminate it, hoping to reduce the negative impact of this phenomenon on society.

\section{ANALYSIS OF NEGATIVE AND TYPICAL STEREOTYPED IMAGE}

Although we all believe that we are in an era of unprecedented fairness, stereotypes (dominant and implicit) affect our behavior every moment and bring many obvious effects to the society:

According to the official data released by the United States in 2014: African-American girls accounted for $12 \%$ of all suspensions, which is the highest among women of all races and ethnicities0. The United States describes this phenomenon as "school pushout", which means there are many negative factors existing in school that prevent students who are suffering the prejudice from completing their studies or being a good student.

Minority students and women have widely received negative stereotypes in the field of mathematics, which not only leads to their performance in mathematics is often lower than their SAT scores predicted scores, but also their anxiety about mathematics is higher than average. Similar phenomena are widespread in Asia, for example, in South Korea, although gender gaps are basically non-existent in the scientific achievements of schools, they are very large in science-related occupations, and only a few female students will incorporate science-related jobs into their career plans.

Extensive notorious stereotypes are of course harmful to the stigmatized group, but may be beneficial 
to another group. Stereotype improvement, due to the awareness of the performance improvement caused by negative stereotypes of outside groups, summarizes this phenomenon0. For instance, when mathematical stereotypes against ethnic minorities and women are widespread, white men tend to perform better in mathematics.

Many scientists have explored the reasons for the formation of stereotypes and the ways in which they have impacts:

Janel A. Georg (2015) has done extensive and comprehensive research on the phenomenon of " school pushout" . He believes that the high misconduct rate of African-American students (especially African-American female students) cannot be explained by the common sense that "blacks are inherently weaker in obedience". In fact, data investigations show that there is no evidence that differences in racial discipline can be explained. Explain the apparently high rate of misconduct among African-American students0. This phenomenon is caused by the school' $s$ excessively strict punishment policy, excessive reliance on disciplinary management of students, and the school' $\mathrm{s}$ systemic racial prejudice system. School administrators, or social stereotypes, originated from slavery. In order to justify the sexual and labor abuse of African women, people describe African women as hypersexual, vulgar, and confrontational. Slavery perpetuates the social belief of "black inferiority, white self-confidence", and it has repeatedly appeared in the country's education system 0 . The near-absolute authority of school administrators in schools has led to school disciplines filled with racial and gender biases from administrators, which in turn strengthened the stereotypes of the younger generation and exerted a huge impact on African-American women.

Steven J. Spencer believes that the main hazard of stereotype influence comes from the threat of stereotypes, that is, when the stigmatized group is in a situation where behavior may be explained by negative stereotypes, additional pressure will make their performance worse.

Spencer analyzed the multiple mediations of stereotype threats from three perspectives: extra stress, threats to self-concepts, and stereotype initiation0. First of all, the extra stress will cause people who experience stereotypes to study harder, and will have extra fear and self-doubt when facing the test. These self-doubts will consume working memory, and people need working memory to improve concentration during the test. And collect information. For activities that do not rely heavily on attention (such as tennis), stereotype threats can cause people to allocate extra attention, which can lead to failure. Secondly, stereotypes can threaten people's self-concepts. People will lower their self-expectations through self-impediment (such as not studying hard and not reading reports), and protect themselves from stereotypes through poor performance of non-reaction ability. Finally, when the stereotype is activated, behavior consistent with the stereotype may automatically occur.

Shin (2015) analyzed the relationship between the average quantity difference brought by gender and the relationship between variables from two aspects: motivation (personal motivation and work motivation) and environment (psychological environment, economic environment and social environment)0. From the point of view of motivation, girls' intrinsic motivation and work motivation are lower than boys. Studies have shown that this phenomenon can be explained by the socialization of gender roles in the family. Girls' parents often think that it is difficult for women to work in science because of stereotypes. Achievement, so reduce the expectation of girls to become scientists. Girls feel this stereotype and internalize it, thus avoiding science-related career plans. From the environmental point of view, the suggestions of important others (friends and family) are effective influencing factors. For teenagers in adolescence, the support from friends will have a direct impact on them. In addition, the common belief from the family is also one of the important influencing factors of career planning and pursuit of scientific career. Parents' positive belief in the importance of science and children's ability will indirectly affect career planning through the influence on self-concept.

Although there are gender differences in most variables, there are gender similarities in the relationships between variables. For example, parents' socioeconomic status (SES) can have a positive impact on motivation and the environment, and this applies to both boys and girls. Higher social status usually means that parents pay more attention to science and provide children with better educational resources. In addition, there is a positive correlation between SES and adolescents' scientific motivation and self-concept.

Walton (2003) and his team did a systematic study on stereotype promotion. They found that people naturally associate negative stereotypes with intelligence tests. For groups affected by negative stereotypes, when conducting related tests They will feel panic, leading to poor performance; for the unaffected groups, the depreciation of outgroups increases their sense of self-efficacy, which is actually beneficial to their performance0. This increase in stereotypes disappears only when the subject is told that the test has nothing to do with gender or race.

There used to be a common sense among both scientists and the public that the more developed the economy is, the more the country promotes gender 
equality, the smaller the occupational gender gap. However, gender paradox is widespread in developed countries. The so-called gender paradox means that in more equal and more developed countries, the gender segregation of different occupations is more obvious. Thomas Breda explained this problem from three aspects: First, although male supremacy has declined to vary degrees in all countries in the past few decades, gender essentialism has not changed. The latter describes males and females as being genetically different but not unequal, which implies that males are better at mathematics may be determined by genes. Secondly, richer countries have developed more liberal individualism and paid more attention to self-expression, which may cause residents to return to the old cultural framework because they are too free. In order to have a clear self-concept, residents will rely on a deep-rooted cultural background. Finally, in developed countries, work is not only a means of earning a living, but also an important tool for self-expression. People will use stereotypes of occupation and gender as a way of emphasizing their self-concepts. Therefore, free career choices provide a greater living space for stereotypes 0 .

After extensive and in-depth research on stereotypes, the researchers found that stereotypes are not limited to one-dimensional negative stereotypes. For example, negative stereotypes of women lead to poor academic performance in mathematics. Stereotypes are in fact widespread and common. No matter which social group, they may be stereotyped by outgroups. For example, in schools, many teachers have the stereotype that "boys have lower reading ability than girls0" ; American society generally thinks that "The stereotype that white men are not good at expressing feelings" . Cuddy (2009) proposed a multi-dimensional Stereotype Content Model (SCM) and found that humans tend to associate perceptual characteristics with social structure. Ability and enthusiasm are not the only perceptual dimensions, but they are indeed core and theoretically meaningful, An important predictable dimension. Based on these two dimensions, SCM can be divided into four categories (high enthusiasm, high ability; high enthusiasm, low ability; low enthusiasm, high ability; low enthusiasm, low ability). Normally, many outside groups will receive conflicts. Stereotypes, such as in the United States, Asians and some outstanding minorities are considered "competitive but indifferent" 0 . Not only that, SCM also exists across cultures, no matter what cultural ideology (individualism or collectivism), it will evaluate the social population through these two dimensions. After analyzing data from six non-US countries, the researchers reported that the stereotypes of older people are warm but incompetent in different cultures 0 .
In fact, scientists' research on stereotypes has gradually shifted from young people to the elderly0. Levy has done a very in-depth investigation and research on the stereotypes of the elderly. She found that when stereotypes are assimilated with surrounding cultures to impress self-concept The function and health of the individual are affected, reflecting the concretization of stereotypes0. It is generally believed that aging is an irresistible process in which the body' $\mathrm{s}$ physical and psychological adaptability to the environment gradually decreases. In fact, those with more positive age stereotypes exhibit better physical functions in their later years0.

\section{CONCLUSION}

In general, this paper summarizes several typical manifestations of stereotypes and scientists' explanations for the reasons behind the phenomena. Stereotypes threaten the lives of many people. We should actively face this problem and create a more equal and safer environment for future generations. Therefore, this article makes several suggestions

1. Reasonable system. Studies have shown that if two groups receive institutional support in a non-competitive environment and have equal contact with each other, the impact of stereotypes can be reduced.

2. Training of teachers. Teachers should be encouraged to resist stereotypes (whether girls are not good at math or boys are not good at reading), because teachers have broad discretion in schools. Under normal circumstances, it is difficult for outsiders (such as the government and courts) to participate in school policy decisions. Therefore, the fair education system relies heavily on teachers to consciously resist stereotypes.

3. Equal treatment. Data shows that women often do not receive the same level of initial support in their academic careers 0 , and institutions tend to provide financial support for men, which makes their subsequent academic careers often inferior to men. Therefore, equal treatment contributes to the advancement of women in academic achievement.

This paper has limitations : the author mentions more negative one-dimensional stereotypes, mainly including women and minorities, rather than multi-dimensional stereotype content models, and does not provide an in-depth introduction to this aspect. At the same time, the research on groups such as men, young men, and the elderly is not thorough enough, and the various groups in society are not comprehensively considered. This is because the paper lacks some universality. Finally, the recommendations in this article are not comprehensive enough, mainly in the academic field (schools and scientific research institutions), and do not involve policy recommendations. 


\section{ACKNOWLEDGMENTS}

This work was supported by Professor Dense Szucs, who is very kind to the students and provides me with a lot of help. I am very grateful to him for his care and encouragement throughout the research process (especially in the topic selection of the thesis).

Additionally, I would like to deeply thank our teaching assistant Yilin Wang of Nanyang Technological University. It is precisely because of his selfless and high-quality teaching that I can absorb so much professional knowledge in a short period and successfully write this paper.

\section{REFERENCES}

[1]Bolukbasi, Tolga, et al. "Man is to computer programmer as woman is to homemaker? debiasing word embeddings." Advances in neural information processing systems 29 (2016): 4349-4357.

[2]Carr, Phyllis L., et al. "Gender differences in academic medicine: retention, rank, and leadership comparisons from the National Faculty Survey." Academic medicine: journal of the Association of American Medical Colleges 93.11 (2018): 1694.

[3]U.S. DEP'T OF EDUC., OFFICE FOR CIVIL RIGHTS, supra note 12 , at 1.

[4]Walton G M, Cohen G L. Stereotype lift [J]. Journal of Experimental Social Psychology, 2003, 39(5): 456-467.

Skiba et al., supra note 10, at 1088 (school 的 52)

[5]George, Janel A. "Stereotype and school pushout: Race, gender and discipline disparities." Ark. L. Rev. 68 (2015): 101.

[6]Spencer, Steven J., Christine Logel, and Paul G. Davies. "Stereotype threat." Annual review of psychology 67 (2016): 415-437.

[7]Shin, Jongho, et al. "Home and motivational factors related to science-career pursuit: Gender differences and gender similarities." International Journal of Science Education 37.9 (2015): 1478-1503.

[8]Breda, Thomas, et al. "Gender stereotypes can explain the gender-equality paradox." Proceedings of the National Academy of Sciences 117.49 (2020): 31063-31069.

[9]Retelsdorf, J., Schwartz, K., \& Asbrock, F. (2015). "Michael can't read!" Teachers' gender stereotypes and boys' reading self-concept. Journal of Educational Psychology, 107(1), 186.
[10]Cuddy, Amy JC, et al. "Stereotype content model across cultures: Towards universal similarities and some differences." British Journal of Social Psychology 48.1 (2009): 1-33.

[11]Cuddy, Amy JC, Michael I. Norton, and Susan T. Fiske. "This old stereotype: The pervasiveness and persistence of the elderly stereotype." Journal of social issues 61.2 (2005): 267-285.

[12]Levy, B. (2009). Stereotype embodiment: A psychosocial approach to aging. Current directions in psychological science, 18(6), 332-336.

[13]Wurm, S., Tesch-Römer, C., \& Tomasik, M. J. (2007). Longitudinal findings on aging-related cognitions, control beliefs, and health in later life. The Journals of Gerontology Series B: Psychological Sciences and Social Sciences, 62(3), P156-P164. 